\title{
Fast neutral lithium beam probing of the edge region of the spherical tokamak ETE
}

\author{
R. M. Oliveira, ${ }^{a)}$ M. Ueda, and L. A. Berni \\ Associated Plasma Laboratory, National Institute for Space Research, 12227-010, São José dos Campos, \\ SP, Brazil \\ H. Iguchi \\ National Institute for Fusion Science, Toki 509-5292, Japan
}

(Presented on 19 April 2004; published 4 October 2004)

\begin{abstract}
The close relationship between the behavior of the edge plasma characteristics and the global confinement in discharges in tokamaks has been extensively explored in recent years. A $15 \mathrm{keV}$ lithium beam with $80 \%$ neutralization efficiency, using a high ion current density emitter and a modified Pierce gun design for extraction of ions, was developed for edge studies on the spherical tokamak ETE- (major radius $R=0.30 \mathrm{~m}$, aspect ration $A=1.5$, designed plasma current of $200 \mathrm{kA}$ with a torodial magnetic field up to $0.4 \mathrm{~T}$ ). Already in the calibration phase this diagnostics confirmed to be an efficient experimental tool, allowing accurate measurements of the fast varying pressure of the discharges in ETE. The measurement of the temporal evolution of the density of the plasma edge with fast neutral lithium beam is in accordance with data from Langmuir probe for the region near the limiter and the one made by the Thomson scattering for the inner region. (c) 2004 American Institute of Physics. [DOI: 10.1063/1.1784534]
\end{abstract}

\section{INTRODUCTION}

As an alternative to the conventional tokamaks, the spherical tori, that are low aspect ratio devices which could lead to compact and low-cost fusion power plants, have as the main advantage the possibility of operation with a high ratio of plasma pressure to magnetic field pressure. ${ }^{1}$ The research program of the spherical tokamak ETE is devoted to the study of operating regimes, confinement properties and current drive schemes. ${ }^{2}$ In the present phase of development of ETE, only inductively heated plasmas have been studied. As a next step, the study of improved confinement regimes has been planned, with the aid of auxiliary heating methods. In order to fulfill the planned scientific schedule for the ETE, the investigation of the edge region of its discharge is mandatory.

It is well known that plasma boundary of tokamaks is characterized by steep gradients of the plasma parameters and by the emission of impurity atoms from the walls and limiters. ${ }^{3}$ Edge fluctuation phenomena are characterized by frequencies of up to $100 \mathrm{kHz}{ }^{4}$ So, an effective probing of this region can only be done if good time and spatial resolutions can be achieved.

Due to the inherent characteristics of allowing high spatial and temporal resolution during the entire discharge lifetime without perturbation of the plasma, ${ }^{5}$ the fast neutral lithium beam (FNLB) diagnostics is being applied to probe the edge region of ETE discharges. However, high energy FNLB devices are capable of deeper probing of a plasma, but with the disadvantage of worst spatial resolution. When

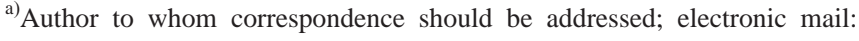
rogerio@plasma.inpe.br
}

lower energy devices are used, the signal-to-noise ratio becomes weak and in general a phase sensitive detection system must be used to improve this relation, which implies a loss of temporal resolution of the measurement. To combine the desirable characteristics of good spatial and temporal resolution during measurements, FNLB diagnostics must produce intense ion beam even with relatively low energy accelerating sources. This article show some important modifications made in the ion source and in the ion gun of a 10-15 keV neutral lithium beam device that made it possible to probe the edge region of ETE discharge with good spatial and temporal resolution. Also, the measurement of the fast varying pressure into ETE vacuum chamber when piezoelectric valves are used for injection of gas, performed with FNLB, are reported here.

\section{EXPERIMENTAL SETUP (FNLB)}

Experimental apparatus of the FNLB diagnostics can be seen in Fig. 1. A modified Pierce gun coupled to three electrostatic cylindrical lenses is being used to accelerate and to focus the ion beam. ${ }^{6} \mathrm{Li}$ ions are emitted through the heating of $\beta$-eucryptite compound ${ }^{7}\left(\mathrm{Li}_{2} \mathrm{O} \cdot \mathrm{Al}_{2} \mathrm{O}_{3} \cdot 2 \mathrm{SiO}_{2}\right)$ to temperature over $1100^{\circ} \mathrm{C}$ in a self-heated molybdenum crucible of $6 \mathrm{~mm}$ diameter and with a $2 \mathrm{~mm}$ depth receptacle. Deflection plates situated straight in front of the cylindrical lenses are used to made small corrections on the trajectory of the beam. Neutralization system is based on a pulsed vapor sodium chamber. For $15 \mathrm{keV}$ lithium ions, $80 \%$ of the neutralization is obtained by charge exchange process. The optical detection system is composed of an adjustable camera lens focused onto the tip of a $7 \mathrm{~m}$ fiber bundle, connected to plano-convex lens, 2 interference filter in cascade [full width 


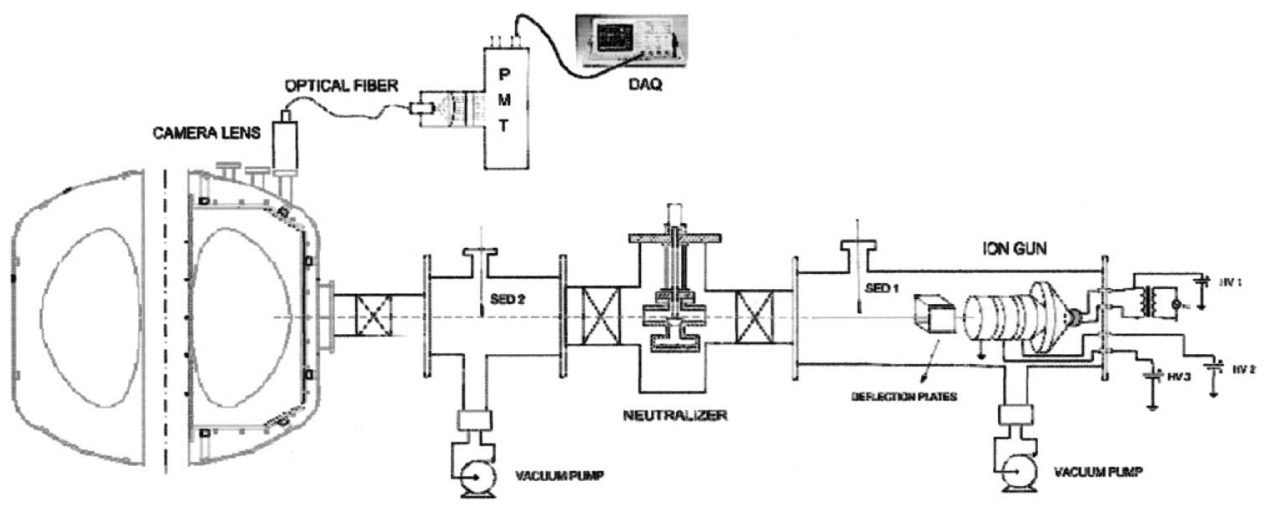

FIG. 1. Schematic drawing of the experimental apparatus (not in scale).

at half maximum (FWHM) $=3 \mathrm{~nm}$ ] and photomultiplier tube. The output signals, presently captured in a digital oscilloscope, will be acquired by the data acquisition system of ETE, ${ }^{8}$ based on VME bus.

\section{IMPROVED ION CURRENT PROPERTIES}

Initial configuration of the ion gun used on the FNLB diagnostics of ETE was similar to the one used in Nagoya, ${ }^{9}$ based on the design of high-current ion optics of a planar Pierce gun, ${ }^{10,11}$ where a linear trajectory of the beam is expected for the source emitting under Child-Langmuir condition. A divergence of the beam exists, however, due to the presence of an orifice of 6.5 -mm-diam on the anode of the gun to accommodate the emitter area of the ion source. This divergence, directly related to the perveance of the gun, ${ }^{12}$ was monitored on the region of the neutralizer by a movable thin copper wire, capable of indicating the profile of the beam.

The performance of the gun regarding the divergence of the beam was simulated by the KARAT, ${ }^{13}$ a particle-in-cell code that can simulate the creation and acceleration of the ion beam, showing its position and size on the trajectory, according to the variation of the distance cathode-anode, changing of geometry of the cathode/anode, and polarization of the electrostatic cylindrical lenses. Best ion collimation was obtained when the angle formed between the cathode surface and the edge of the beam changed from $67.5^{\circ}$ (Pierce angle) to $60^{\circ}$. A new gun was built taking into account this modification. Figure 2 shows a comparison between experimental results of the ion emission profile for the Nagoyatype gun and for its modified version, where a decrease on the FWHM of about $1 / 2 \mathrm{~cm}$ occurred in favor of the last one.

A tenfold increase of the $\mathrm{Li}^{+}$emission of $\beta$-eucryptite compound was also obtained after its transformation from pasty to glassy form. ${ }^{14}$ Comparative results among this stable and long-lived ion source with commonly used paty sources and commercial loads are described in a previous article. ${ }^{15}$ The work function potential at the surface of the glassy compound was found to be $6 \%$ lower than in the case of the powder counterpart. Current density of $1 \mathrm{~mA} / \mathrm{cm}^{2}$ was attained by this load when heated to $1200^{\circ} \mathrm{C}$ (input heating power of abount $40 \mathrm{~W}$ ). Lifetime of this glassy $\beta$-eucryptite allows ion extraction at necessary current level for more than $5 \mathrm{~h}$ in continuous operation, against less than $1 \mathrm{~h}$ for the commercial load.
The changes performed in the ion gun and in the ion source were confirmed to be efficient which makes it possible to probe of the edge of the plasma in ETE with FNLB, without being necessary to use the phase sensitive detection system. It will provide temporal resolution during measurements dictated by the time scale of the atomic physics processes and by the photon flux detected. For acceleration voltage of $10-15 \mathrm{kV}$, the product of beam atom speed and the lifetime of the excited level $(\operatorname{Li}[2 p-2 s])$ varies from 1.3 to $1.7 \mathrm{~cm}$, respectively. Due to collisions suffered by $\mathrm{Li}$ atoms in the $2 p$ state leading to excitation to higher levels, deexcitation and ionization, the effective spatial resolution is less than $1 \mathrm{~cm}$ for both cases.

\section{RESULTS AND DISCUSSION}

\section{A. Fast varying pressure measurements with FNLB probe}

During the calibration phase, Li beam was injected into the ETE chamber which was filled with hydrogen at different pressures. The intensity of the light collected by the optical detection system coming from the $\mathrm{Li}$ resonance line $(6708 \AA)$, increased for pressures ranging from 4 $\times 10^{-6}$ Torr to $2 \times 10^{-3}$ Torr. So, a direct ratio between the intensity of the output signal of the photomultiplier and working pressure in ETE was established. This relation was used to monitor the fast varying pressure in vacuum chamber when the gas was fed by the puff valves during discharges. The graph of Fig. 3(a) demonstrates the variation on the

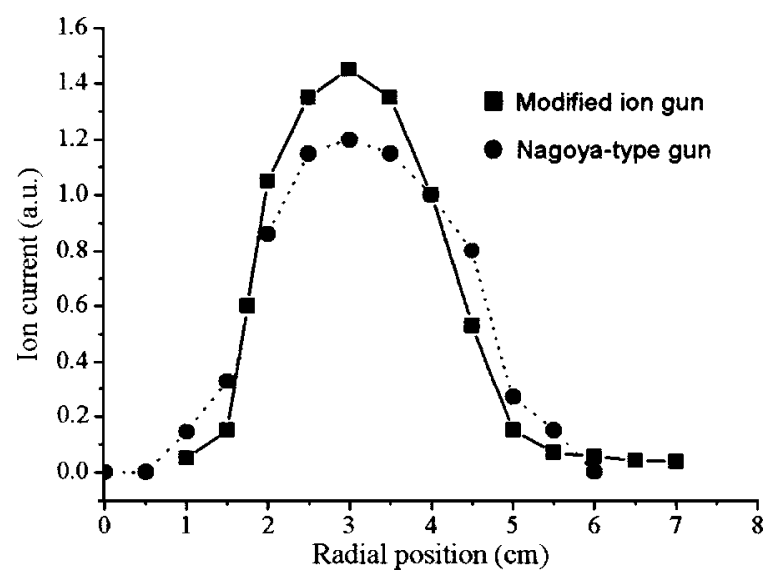

FIG. 2. Beam profile measurement for Nagoya-type gun and its modified version. 

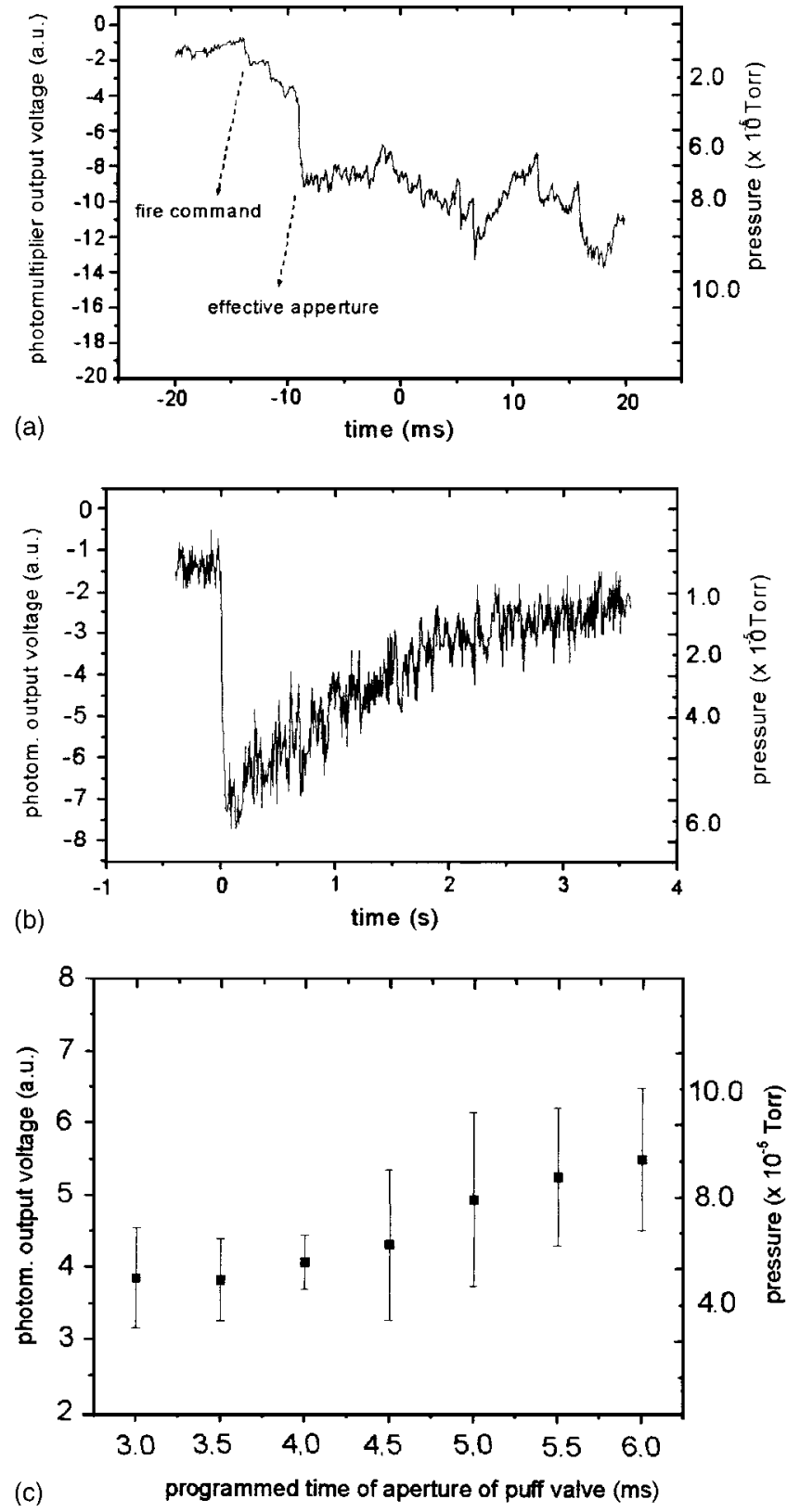

FIG. 3. Monitoring of the fast varying pressure inside vacuum chamber: (a) by setting the time of aperture of the puff valve to $5 \mathrm{~ms}$, (b) after its filling and evacuation by vacuum system, (c) by varying the time of aperture of the piezoelectric valve of injection of gas.

intensity of the output signal of the photomultiplier by puffing of the gas into the vacuum chamber, being the time of aperture of the valve settled to $5 \mathrm{~ms}$. There it is possible to observe the time delay between the firing of the valve and its effective opening, which is of approximately $6 \mathrm{~ms}$. After that, a pressure of about $8 \times 10^{-5}$ Torr is established in the vacuum chamber. Figure 3(b) shows the exact instant of the fast input of gas into the chamber and consequent reduction of the pressure due to vacuum pumping. It takes about $4 \mathrm{~s}$ after the input of the gas until it has been completely evacuated by vacuum system. Several measurements were done by puffing gas in the ETE chamber by changing the time of aperture of the valve. The graph of Fig. 3(c) shows the medium pressure value obtained for each case with respective error bar. Here, radial resolution is about $2 \mathrm{~cm}$, since there is no effect of the plasma shortening the lifetime of the excited state of lithium atoms.

\section{B. Measurements at the plasma edge}

The measurement of the temporal evolution of the density $n_{e}$ of the plasma edge with FNLB is in accordance with data from the Langmuir probe for the region near the limiter and the one made by the Thomson scattering for the inner region.

Plasma density measured by Langmuir probe for the region near the limiter $(R=55 \mathrm{~cm})$ is in the range of $2-3$ $\times 10^{17} \mathrm{~m}^{-3}$; for the inner region of the edge plasma $(R$ $=48 \mathrm{~cm})$ typical Thomson scattering measurements are of $2-3 \times 10^{18} \mathrm{~m}^{-3}$. Temperatures ranged from 10 to $30 \mathrm{eV}$. While probing this region, attenuation of the Li beam can be neglected. In fact, through Eq. (1), which defines the attenuation of local density of the neutral $\mathrm{Li}$ beam $n_{b}(r)$ in its path, ${ }^{16}$ less than $4 \%$ of the beam is lost until it reaches the radial position of $R=48 \mathrm{~cm}$, that is $7 \mathrm{~cm}$ away from the limiter. For this case it was considered in the calculation of an attenuation cross section $\sigma_{T}=4 \times 10^{-15} \mathrm{~cm}^{2}$. This value is the sum of cross sections for ionization and charge transfer of the lithium beam. ${ }^{17}$

$$
n_{b}(r)=n_{b}(0) \exp \left\{-\frac{\langle\sigma v\rangle_{i}}{\nu_{b}} \int_{0}^{r} n_{e}(r) d r\right\},
$$

where $\langle\sigma v\rangle_{i}$ and $\nu_{b}$ are the effective rate coefficient for ionization and the beam velocity, respectively.

No significant variation on the cross section for excitation of $\mathrm{Li}$ atom was observed for a $10-15 \mathrm{keV}$ lithium beam interacting in a plasma with temperature ranging from 10 to $30 \mathrm{eV}$. While the conditions of low attenuation of the $\mathrm{Li}$ beam, low sensitivity of the excitation rate for the $\mathrm{Li}[2 s$ $-2 p$ ] transition with $T_{e}$, and low beam velocity are obeyed for edge region of ETE plasma, local electron density in this case is proportional to the intensity of the resonance line radiation $\mathrm{Li}[2 s-2 p]$ at $6708 \AA$, due to the interaction of the beam with the electrons of the plasma. In fact, the principle for density determination used for this situation is based on the comparison of the photon flux profiles emitted from the lithium atoms due to interaction with plasma electrons $N_{v p}$ and due to the interaction with hydrogen gas $N_{v g}$, under same discharge conditions, according to the relation below ${ }^{6}$ :

$$
n_{e}(r)=\frac{N_{v p}(r)}{N_{v g}} \frac{Q_{g}}{Q_{p}} n_{g},
$$

where $Q_{g}$ and $Q_{p}$ are the excitation cross section for collision of the beam atoms with neutral atoms in the gas and the effective excitation cross section, respectively.

Presently, the energy delivered by the toroidal and ohmic heating capacitor banks represent just $1 / 4$ of the total energy expected to be delivered, in order to attain projected conditions of operation in ETE. Plasma current of $200 \mathrm{kA}$ will be reached and densities of the order of $10^{19} \mathrm{~m}^{-3}$ will also be attained, even in the edge region. Lithium beam attenuation will be stronger for this case and density reconstruction code will be used to recover density profiles. ${ }^{18}$ 

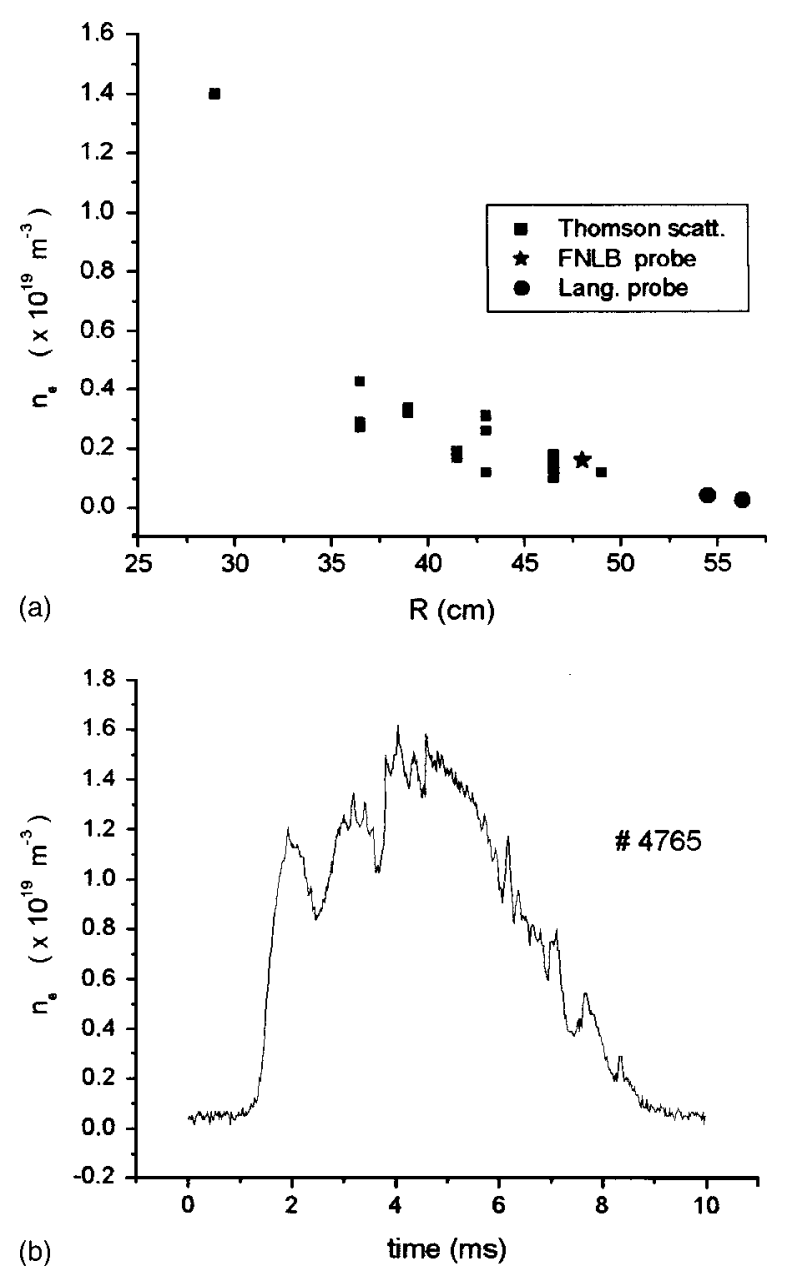

FIG. 4. Experimental measurements: (a) radial profile of the density in ETE measured by Thomson scattering, fast neutral lithium beam probe and Langmuir pobe, (b) temporal evolution of the edge density in ETE discharge $(R=48 \mathrm{~cm})$.

Radial profile of the density in ETE has been obtained by measurements made by Thomson scattering $(R=30 \mathrm{~cm}$ to $R=46.5 \mathrm{~cm})$, FNLB probe $(R=48 \mathrm{~cm})$, and Langmuir probe $(R=53-55 \mathrm{~cm})$, as is shown in Fig. 4(a). These experimental data were obtained for shots $4732-4779$ for time $t=5 \mathrm{~ms}$. Time evolution of the density for $R=48 \mathrm{~cm}$ was obtained by measurement with FNLB diagnostics, as can be shown in Fig. 4(b) for shot 4765. The actual configuration of FNLB and Thomson scattering diagnostics allows the acquisition of the density of only one radial position by shot. In the near future both systems will be upgraded. A multipoint Thomson scattering based on time-delay technique ${ }^{19}$ will allow the measurement of density profiles with ten spatial points per laser shot, per polychromator. FNLB will use a flexible multichannel photomultiplier, composed of an array of $8 \times 8$ independent cells. In the best case, no cross talk and enough intensity of the signal, up to 64 radial positions of the plasma can be swept with this experimental arrangement of the optical detection system. Initially, through convenient lens configuration, the light from eight radial positions of the plasma, conducted by a set of eight optical fibers, will be focused onto eight cells of the photomultiplier, and a density profile will be obtained in a single shot.

In spite of background radiation, mainly due to $\mathrm{H}_{\alpha}$ emission, high spatial and temporal resolutions in measurements with the actual configuration of FNLB diagnostics is being obtained for ETE discharges. Modifications performed in the ion gun and in the ion source were crucial in this phase. Good conditioning of the machine through glow discharges was also very important to eleiminate the tail of $\mathrm{H}_{\alpha}$ emission and to improve signal-to-noise ratio during FNLB measurements. Experimental results show good agreement with data from Thomson scattering for the inner region of the plasma and with Langmuir probe data for the region near the limiter. The increase on the number of points to be observed with FNLB probe will allow the study of the physics of the edge region of a spherical tokamak, with such a diagnostics.

${ }^{1}$ Y.-K. M. Peng and D. J. Strickler, Nucl. Fusion 26, 769 (1986).

${ }^{2}$ G. O. Ludwig et al., Braz. J. Phys. 33, 848 (2003).

${ }^{3}$ E. Hintz, Trans. Fusion Technol. 25, 333 (1994).

${ }^{4}$ M. Bruchhausenet al., Plasma Phys. Controlled Fusion 46, 489 (2004).

${ }^{5} \mathrm{H}$. Iguchi and K. Kadota et al., Rev. Sci. Instrum. 56, 1050 (1985).

${ }^{6}$ R. M. Oliveira et al., Braz. J. Phys. (to be published).

${ }^{7}$ J. P. Blewet and E. J. Jones, Phys. Rev. 50, 464 (1936).

${ }^{8}$ R. M. Oliveira et al., Fusion Eng. Des. 60, 403 (2002).

${ }^{9}$ M. Ueda and H. Iguchi et al., J. Nucl. Mater. 196-198, 923 (1992).

${ }^{10}$ G. R. Brewer, Focusing of Charged Particles, edited by A. Septier (Academic, New York, 1967), Vol. II.

${ }^{11}$ J. R. Pierce, Theory and Design of Electron Beams (Van Nostrand, New York, 1954).

${ }^{12}$ D. M. Thomas and W. P. West, Rev. Sci. Instrum. 59, 1735 (1988).

${ }^{13}$ V. P. Tarakanov, User's Manual for Code Karat (Berkeley Research Associates, 1997).

${ }^{14}$ M. Ueda et al., J. Phys. D 30, 2711 (1997).

${ }^{15}$ R. M. Oliveira et al., Jpn. J. Appl. Phys., Part 1 43, 1154 (2004).

${ }^{16}$ T. Morisaki and A. Komori et al., Rev. Sci. Instrum. 74, 1865 (2003).

${ }^{17}$ K. Kadota and K. Tsuchida et al., Plasma Phys. 20, 1011 (1978).

${ }^{18} \mathrm{P}$. Bogen and E. Hintz, in Physics of Plasma-Wall Interaction in Controlled Fusion, edited by D. E. Post and R. Behrish (Plenum, New York, 1986), p. 211.

${ }^{19}$ L. A. Berni et al., Braz. J. Phys. (to be published). 